\title{
Local Maximum Likelihood Estimation and Inference
}

\author{
Jianqing Fan \\ Department of Statistics, University of North Carolina, Chapel Hill, NC 27599-3260, USA, and \\ Department of Statistics, University of California, Los Angeles, CA 90095-1555, USA
}

Mark Farmen

Ross Products Division, Abbott Laboratories, Columbus OH 43215, USA

Irène Gijbels

Institut de Statistique, Université Catholique de Louvain, Voie du Roman Pays 20, B-1348

Louvain-la-Neuve, Belgium

October 15, 1998

\begin{abstract}
Local maximum likelihood estimation is a nonparametric counterpart of the widely-used parametric maximum likelihood technique. It extends the scope of the parametric maximum likelihood method to a much wider class of parametric spaces. Associated with this nonparametric estimation scheme is the issue of bandwidth selection and bias and variance assessment. This article provides a unified approach to selecting a bandwidth and constructing confidence intervals in local maximum likelihood estimation. The approach is then applied to least-squares nonparametric regression and to nonparametric logistic regression. Our experiences in these two settings show that the general idea outlined here is powerful and encouraging.
\end{abstract}

\section{Introduction}

Maximum likelihood estimation provides a useful blueprint for various statistical estimation problems. It also provides a unified method for constructing confidence intervals for parameters. A

Abbreviated title: Local likelihood estimation.

AMS 1991 subject classification. Primary 62G07. Secondary 62A10.

Key words and phrases: Bandwidth selection; confidence intervals; generalized linear models; logit regression; maximum likelihood; nonparametric regression. 
drawback of this method is that one has to assume a particular parametric form for the unknown target function. This restrictive assumption can be removed by using a maximum local kernelweighted likelihood estimator. An important issue is then to choose the size of the neighborhood. Further, the question arises of how to construct confidence intervals. These problems are challenging and so far they have no satisfactory answer in the literature. This article attempts to provide a unified approach to these problems.

Local maximum likelihood estimation is based on the idea of local fitting. Scatterplot smoothing by local fitting has been around for many years. Local fitting is indeed a particular useful technique in nonparametric estimation. Among the earlier papers in the context of nonparametric regression are Stone (1977, 1980), Cleveland (1979) and Friedman and Stuetzle (1981). Further, there is a vast literature on likelihood-based models in various domains of application, and these models mainly appear in parametric estimation problems.

The idea of using local fitting for likelihood-based regression models was applied by Tibshirani and Hastie (1987) to the class of generalized linear models (see Nelder and Wedderburn (1972)) and to the proportional hazards model of Cox (1972). Fan, Heckman and Wand (1995) show that the approach has good sampling properties when used with local polynomial fitting in the context of generalized linear models. Staniswalis (1989) approached the same problem using a kernel method. These nice sampling properties carry further to the hazard regression setting (see Fan, Gijbels and King (1997)). There is a vast interest in applying the local likelihood method to the problem of density estimation or hazard rate estimation. For more on this, see for example Hjort $(1991,1995)$, Jones (1994), Copas (1995), Hjort and Glad (1995), Hjort and Jones (1996) and Loader (1996). A related idea to the local maximum likelihood method is the local estimating equation approach introduced by Carroll, Ruppert and Welsh (1996) who uses the empirical-bias idea of Ruppert (1995) to select the bandwidth.

An important issue when using local techniques is the determination of the 'local neighborhood', which is commonly described by a kernel function $K$ and a bandwidth parameter $h$. It is wellknown that among these two quantities, the choice of the bandwidth is the more crucial one. This bandwidth controls the size of the local neighborhood, and can be chosen to be constant or to depend on location. 
The building blocks for bandwidth selection are bias and variance estimates of the nonparametric estimator. A general idea for this estimation task is proposed in the paper, and is applicable to most of the likelihood-based models. The idea is an extension of the pre-asymptotic substitution method developed by Fan and Gijbels (1995) in the least-squares context. The bias assessment relies on the difference of two maximum local likelihood fits with different accuracies. Fan and Gijbels (1995) provide extensive evidence showing that the resulting procedure performs very well and we show further in this paper that the generalized idea is appealing for logistic regression. Therefore, it is expected that the extension will work well in a more general likelihood context.

The assessed bias and variance also have important applications in constructing confidence intervals and even confidence bands. Indeed, one can use the estimated bias and variance and rely on the asymptotic normality of the estimator to construct confidence intervals and confidence bands. See for example Eubank and Speckman (1993).

The organization of the paper is as follows. In the next section we discuss briefly the idea of local likelihood techniques using local polynomial fitting. Sections 3 and 4 show how to access the bias and variance of the local maximum likelihood estimator. Section 5 discusses a simple approach to select a pilot bandwidth. The applications to bandwidth selection are discussed in Section 6 . Section 7 presents how to construct confidence intervals based on the assessed bias and variance. The proposed methodology is then illustrated for local least-squares regression and for local logistic regression in Section 8.

\section{Local log-likelihood estimation}

In order to introduce the local likelihood idea, we recall the maximum likelihood estimation method for a parametric model. Suppose that the $i^{\text {th }}$ observation $\left(X_{i}, Y_{i}\right)$ in a sample $\left(X_{1}, Y_{1}\right), \cdots,\left(X_{n}, Y_{n}\right)$ has a contribution $\ell\left\{g\left(X_{i}\right), Y_{i}\right\}$ to the conditional log-likelihood, where $g(\cdot)$ is an unknown parametrized function of interest, i.e. $g(x)=g_{\theta}(x)$ where $\theta$ is an unknown parameter. The conditional log-likelihood of the $n$ observations is then given by $\sum_{i=1}^{n} \ell\left\{g_{\theta}\left(X_{i}\right), Y_{i}\right\}$. We require that $\theta$ is the unique solution to the likelihood equation

$$
E\left\{\ell^{\prime}\left\{g_{\theta}(x), Y\right\} \mid X=x\right\}=0
$$


where $\ell^{\prime}(t, u)=\frac{\partial}{\partial t} \ell(t, u)$. Note that one can regard (2.1) as the definition of the parameter $\theta$.

We now turn to nonparametric estimation of $g(\cdot)$ in which the form of $g(\cdot)$ is completely unknown. Suppose that we want to estimate $g\left(x_{0}\right)$. Assume that the function $g$ has a $(p+1)^{t h}$ continuous derivative at the point $x_{0}$. For data points $X_{i}$ in a neighborhood of $x_{0}$ we approximate $g\left(X_{i}\right)$ via a Taylor expansion by a polynomial of degree $p$ :

$$
g\left(X_{i}\right) \approx g\left(x_{0}\right)+g^{\prime}\left(x_{0}\right)\left(X_{i}-x_{0}\right)+\cdots+\frac{g^{(p)}\left(x_{0}\right)}{p !}\left(X_{i}-x_{0}\right)^{p} \equiv \mathbf{X}_{i}^{T} \beta^{0},
$$

where $\mathbf{X}_{i}=\left(1, X_{i}-x_{0}, \cdots,\left(X_{i}-x_{0}\right)^{p}\right)^{T}$ and $\beta^{0}=\left(\beta_{0}^{0}, \cdots, \beta_{p}^{0}\right)^{T}$, with $\beta_{\nu}^{0}=g^{(\nu)}\left(x_{0}\right) / \nu !, \nu=$ $0,1, \cdots, p$. For data points $\left(X_{i}, Y_{i}\right)$ in a neighborhood of $x_{0}$, the contribution to the log-likelihood is $\ell\left(\mathbf{X}_{i}^{T} \beta, Y_{i}\right)$, weighted by $K_{h}\left(X_{i}-x_{0}\right)$, with $K_{h}(\cdot)=K(\cdot / h) / h$ where $\beta=\left(\beta_{0}, \cdots, \beta_{p}\right)^{T}$ is the model parameter. These considerations yield the conditional local kernel-weighted log-likelihood

$$
L_{p}\left(\beta ; h, x_{0}\right)=\sum_{i=1}^{n} \ell\left(\mathbf{X}_{i}^{T} \beta, Y_{i}\right) K_{h}\left(X_{i}-x_{0}\right) .
$$

The subscript $p$ indicates the degree of the polynomial used for the local fitting. Maximizing the local kernel-weighted log-likelihood (2.2) with respect to $\beta$ gives the vector of estimators $\widehat{\beta}=$ $\left(\widehat{\beta}_{0}, \cdots, \widehat{\beta}_{p}\right)^{T}$. Estimators $\widehat{g}_{\nu}\left(x_{0}\right)$ for $g^{(\nu)}\left(x_{0}\right), \nu=0,1, \cdots, p$ are then given by

$$
\widehat{g}_{\nu}\left(x_{0}\right)=\nu ! \widehat{\beta}_{\nu}
$$

For simplicity, (2.2) is also referred to as the local likelihood.

To illustrate the above concept we consider the normal regression model $Y=g(X)+\varepsilon$ with $\varepsilon \sim \mathrm{N}\left(0 ; \sigma^{2}\right)$, and $X$ and $\varepsilon$ independent. The conditional local log-likelihood is

$$
-\log (\sqrt{2 \pi} \sigma) \sum_{i=1}^{n} K_{h}\left(X_{i}-x_{0}\right)-\frac{1}{2 \sigma^{2}} \sum_{i=1}^{n}\left\{Y_{i}-\sum_{j=0}^{p} \beta_{j}\left(X_{i}-x_{0}\right)^{j}\right\}^{2} K_{h}\left(X_{i}-x_{0}\right),
$$

which has to be maximized with respect to $\beta$. This is equivalent to minimizing

$$
\sum_{i=1}^{n}\left\{Y_{i}-\sum_{j=0}^{p} \beta_{j}\left(X_{i}-x_{0}\right)^{j}\right\}^{2} K_{h}\left(X_{i}-x_{0}\right)
$$

leading to local polynomial regression, also referred to as locally weighted least-squares regression.

In the above example, the unknown function was a mean regression function. In other contexts, $g(\cdot)$ will be another unknown function of interest. For example, $g(\cdot)$ can be a transformed conditional mean function in generalized linear models, or the risk contribution function of covariates to 
the conditional hazard function in a proportional hazards model encountered in survival analysis, or the logarithm of the density function in a density estimation problem.

Note that the local kernel-weighted likelihood method is still applicable when the likelihood function involves a constant scale factor such as $\sigma$ in (2.4). Even when $\sigma$ depends on the location $x_{0}$, the local kernel-weighted likelihood method $(2.4)$ can still be used to estimate $g(\cdot)$ because of local homoscedacity: $\sigma^{2}\left(X_{i}\right) \approx \sigma^{2}\left(x_{0}\right)$ for $X_{i}$ in a neighborhood of $x_{0}$. To estimate the scale parameter function $\sigma(\cdot)$ in the latter situation, we can have two possible methods. The first one is to approximate $\log \left\{\sigma^{2}(\cdot)\right\}$ locally by a polynomial function with unknown parameters denoted by $\gamma$ and then maximize (2.4) simultaneously with respect to parameters $\beta$ and $\gamma$. The second approach is to estimate $\beta$ first by regarding $\sigma^{2}(\cdot)$ locally as a constant, and then apply the local modeling idea to $\log \left\{\sigma^{2}(\cdot)\right\}$ by using a different bandwidth. The second approach is basically a residual-based method, which is similar to that given in Ruppert, Wand, Holst and Hössjer (1995) who show that the latter method is effective. While the above discussions are in the context of the normal models, the idea is expandable to the general likelihood setting.

\section{Assessing the bias of the estimator}

In this and in the next section, we focus on how to estimate the bias and variance of the local maximum likelihood estimator. The estimated bias and variance will be used to select the bandwidth and to construct confidence intervals in Sections 6 and 7, respectively.

The bias of the estimator $\widehat{\beta}$ comes from the approximation error in the Taylor expansion. Let $r\left(X_{i}\right)=g\left(X_{i}\right)-\sum_{j=0}^{p} g^{(j)}\left(x_{0}\right)\left(X_{i}-x_{0}\right)^{j} / j$ ! denote this approximation error at the point $X_{i}$. Suppose that the $(p+a+1)^{t h}$ derivative of the function $g$ exists at the point $x_{0}$ for some $a>0$. Then, a further expansion of $g\left(X_{i}\right)$ gives an approximation to the approximation error:

$$
r\left(X_{i}\right) \approx \beta_{p+1}^{0}\left(X_{i}-x_{0}\right)^{p+1}+\cdots+\beta_{p+a}^{0}\left(X_{i}-x_{0}\right)^{p+a} \equiv r_{i}
$$

where $a$ denotes the order of the approximation. The choice of $a$ will have some effect on the performance of the estimated bias. A discussion on the choice of $a$ can be found in Fan and Gijbels (1995). Good practical performance is obtained with $a=2$.

Suppose for a moment that the quantities $r_{i}$ are known. Then, a more precise local log-likelihood 
is

$$
L_{p}^{*}\left(\beta ; h, x_{0}\right)=\sum_{i=1}^{n} \ell\left(\mathbf{X}_{i}^{T} \beta+r_{i}, Y_{i}\right) K_{h}\left(X_{i}-x_{0}\right)
$$

The maximizer of the local log-likelihood $L_{p}^{*}\left(\beta ; h, x_{0}\right)$ will be denoted by $\widehat{\beta}^{*}=\widehat{\beta}^{*}\left(x_{0}\right)$. The bias of $\widehat{\beta}\left(x_{0}\right)$ can then be estimated by $\widehat{\beta}\left(x_{0}\right)-\widehat{\beta}^{*}\left(x_{0}\right)$. However, the computation of $\widehat{\beta}^{*}=\widehat{\beta}^{*}\left(x_{0}\right)$ can be avoided as follows. Let

$$
L_{p}^{* \prime}\left(\beta ; h, x_{0}\right)=\frac{\partial}{\partial \beta} L_{p}^{*}\left(\beta ; h, x_{0}\right) \quad \text { and } \quad L_{p}^{* \prime \prime}\left(\beta ; h, x_{0}\right)=\frac{\partial^{2}}{\partial \beta^{2}} L_{p}^{*}\left(\beta ; h, x_{0}\right)
$$

denote the gradient vector and the Hessian matrix, respectively, of the local log-likelihood $L_{p}^{*}$. Since $\widehat{\beta}^{*}\left(x_{0}\right)$ is the maximizer of $L_{p}^{*}\left(\beta ; h, x_{0}\right)$, a Taylor expansion gives

$$
0=L_{p}^{* \prime}\left(\widehat{\beta}^{*} ; h, x_{0}\right) \approx L_{p}^{* \prime}\left(\widehat{\beta} ; h ; x_{0}\right)+L_{p}^{* \prime \prime}\left(\widehat{\beta} ; h, x_{0}\right)\left\{\widehat{\beta}^{*}\left(x_{0}\right)-\widehat{\beta}\left(x_{0}\right)\right\}
$$

and this leads us to define the estimated bias vector

$$
\widehat{b}_{p}\left(x_{0}\right)=\left\{L_{p}^{* \prime \prime}\left(\widehat{\beta} ; h, x_{0}\right)\right\}^{-1} L_{p}^{* \prime}\left(\widehat{\beta} ; h, x_{0}\right)
$$

To get better insight into the bias approximation (3.3), let us look at the normal likelihood (2.5). Denote by $\mathbf{X}$, the design matrix of the regression problem, i.e. the $n \times(p+1)$ matrix whose $(i, j)^{t h}$-element is $\left(X_{i}-x_{0}\right)^{j-1}$, and let $\mathbf{W}=\operatorname{diag}\left\{K_{h}\left(X_{i}-x_{0}\right)\right\}$ be the diagonal matrix containing the weights. Then, except for a constant factor,

$$
L_{p}^{*}\left(\beta ; h, x_{0}\right)=(\mathbf{y}-\mathbf{X} \beta-\mathbf{r})^{T} \mathbf{W}(\mathbf{y}-\mathbf{X} \beta-\mathbf{r})
$$

where $\mathbf{y}=\left(Y_{1}, \cdots, Y_{n}\right)^{T}$ and $\mathbf{r}=\left(r_{1}, \cdots, r_{n}\right)^{T}$. Further, $\widehat{\beta}=\left(\mathbf{X}^{T} \mathbf{W X}\right)^{-1} \mathbf{X}^{T} \mathbf{W} \mathbf{y}$, and hence

$$
L_{p}^{* \prime}\left(\widehat{\beta} ; h, x_{0}\right)=2 \mathbf{X}^{T} \mathbf{W r} \quad \text { and } \quad L_{p}^{* \prime \prime}\left(\widehat{\beta} ; h, x_{0}\right)=2 \mathbf{X}^{T} \mathbf{W X}
$$

Therefore

$$
\widehat{b}_{p}\left(x_{0}\right)=\left(\mathbf{X}^{T} \mathbf{W X}\right)^{-1} \mathbf{X}^{T} \mathbf{W r}
$$

which is equal to the approximation of the bias, $E(\widehat{\beta} \mid \not X)-\beta^{0}$, obtained in Fan and Gijbels (1995), where $X X$ denotes $\left(X_{1}, \cdots, X_{n}\right)$.

Recall that the approximated bias (3.3) depends on the quantities $r_{1}, \cdots, r_{n}$, which are unknown. These quantities will be estimated by fitting a polynomial of degree $p+a$ locally via (2.2), using a 
pilot bandwidth $h^{*}$. This gives estimates $\widehat{\beta}^{(p+a)}=\left(\widehat{\beta}_{0}, \cdots, \widehat{\beta}_{p+a}\right)^{T}$, which are then substituted into expression (3.1), yielding estimates $\widehat{r}_{1}, \cdots, \widehat{r}_{n}$ of $r_{1}, \cdots, r_{n}$. These estimates are then substituted into (3.2), leading to the estimated bias as in (3.3). Denote the estimated bias of $\widehat{\beta}_{\nu}$ by $\widehat{B}_{p, \nu}\left(x_{0} ; h\right)$, the $(\nu+1)^{t h}$ element of $\widehat{b}_{p}\left(x_{0}\right)$.

The choice of the pilot bandwidth $h^{*}$ will be discussed in Section 5 .

\section{Assessing the variance of the estimator}

To get a grip on the variance, first note that,

$$
0=L_{p}^{\prime}\left(\widehat{\beta} ; h, x_{0}\right) \approx L_{p}^{\prime}\left(\beta^{0} ; h, x_{0}\right)+L_{p}^{\prime \prime}\left(\beta^{0} ; h, x_{0}\right)\left(\widehat{\beta}-\beta^{0}\right) .
$$

This leads to

$$
\widehat{\beta}-\beta^{0} \approx-\left\{L_{p}^{\prime \prime}\left(\beta^{0} ; h, x_{0}\right)\right\}^{-1} L_{p}^{\prime}\left(\beta^{0} ; h, x_{0}\right),
$$

and an approximation for the conditional variance is

$$
\operatorname{Var}(\widehat{\beta} \mid \not X) \approx\left\{L_{p}^{\prime \prime}\left(\beta^{0} ; h, x_{0}\right)\right\}^{-1} \operatorname{Var}\left\{L_{p}^{\prime}\left(\beta^{0} ; h, x_{0}\right) \mid \not X\right\}\left\{L_{p}^{\prime \prime}\left(\beta^{0} ; h, x_{0}\right)\right\}^{-1}
$$

The Hessian matrix $L_{p}^{\prime \prime}\left(\beta^{0} ; h, x_{0}\right)$ can be estimated by $L_{p}^{\prime \prime}\left(\widehat{\beta} ; h, x_{0}\right)$, and the conditional variance on the right-hand side of (4.1) can be approximated as follows. From (2.2) we obtain

$$
\begin{aligned}
\operatorname{Var}\left\{L_{p}^{\prime}\left(\beta^{0} ; h, x_{0}\right) \mid \not \mathbf{X}\right\} & =\sum_{i=1}^{n} \operatorname{Var}\left\{\frac{\partial}{\partial \beta} \ell\left(\mathbf{X}_{i}^{T} \beta, Y_{i}\right) \mid \not \mathbf{X}\right\}_{\beta=\beta^{0}} K_{h}^{2}\left(X_{i}-x_{0}\right) \\
& =\sum_{i=1}^{n} \operatorname{Var}\left\{\ell^{\prime}\left(\mathbf{X}_{i}^{T} \beta^{0}, Y_{i}\right) \mid X_{i}\right\} \mathbf{X}_{i} \mathbf{X}_{i}^{T} K_{h}^{2}\left(X_{i}-x_{0}\right) .
\end{aligned}
$$

Since $X_{i}$ has significant weight only in a neighborhood around $x_{0}$,

$$
\operatorname{Var}\left\{\ell^{\prime}\left(\mathbf{X}_{i}^{T} \beta^{0}, Y_{i}\right) \mid X_{i}\right\} \approx \operatorname{Var}\left[\ell^{\prime}\left\{g\left(x_{0}\right), Y\right\} \mid X=x_{0}\right]
$$

Thus, we have

$$
\operatorname{Var}\left\{L_{p}^{\prime}\left(\beta^{0} ; h, x_{0}\right) \mid \not X\right\} \approx \operatorname{Var}\left\{\ell^{\prime}\left(g\left(x_{0}\right), Y\right) \mid X=x_{0}\right\} \bar{S}_{n},
$$

where $\bar{S}_{n}=\sum_{i=1}^{n} \mathbf{X}_{i} \mathbf{X}_{i}^{T} K_{h}^{2}\left(X_{i}-x_{0}\right)$. Combining (4.1) and (4.2) we obtain the following approximation of the conditional variance of $\widehat{\beta}$ :

$$
\operatorname{Var}(\widehat{\beta} \mid \not X) \approx \Sigma\left(x_{0}\right)=\operatorname{Var}\left\{\ell^{\prime}\left(g\left(x_{0}\right), Y\right) \mid X=x_{0}\right\}\left\{L_{p}^{\prime \prime}\left(\beta^{0} ; h, x_{0}\right)\right\}^{-1} \bar{S}_{n}\left\{L_{p}^{\prime \prime}\left(\beta^{0} ; h, x_{0}\right)\right\}^{-1}
$$


The unknown local parameter $\beta^{0}$ in (4.3) can be estimated by $\widehat{\beta}$. The first factor in (4.3) is also unknown and has to be estimated. We separate this into two cases. The first case is that $\operatorname{Var}\left\{\ell^{\prime}\left(g\left(x_{0}\right), Y\right) \mid X=x_{0}\right\}=V\left\{g\left(x_{0}\right)\right\}$ for some known function $V(\cdot)$ such as for the Bernoulli, Poisson and Exponential distributions in the context of generalized linear models. In this case, we estimate the conditional variance by $V\left\{\widehat{g}_{0}\left(x_{0}\right)\right\}$. The second case is that in which we do not have such a form. The normal likelihood model is an example. By (2.1), it follows that

$$
\operatorname{Var}\left\{\ell^{\prime}\left(g\left(x_{0}\right), Y\right) \mid X=x_{0}\right\}=E\left[\left\{\ell^{\prime}\left(g\left(x_{0}\right), Y\right)\right\}^{2} \mid X=x_{0}\right]
$$

This quantity can be estimated by

$$
\frac{\sum_{i=1}^{n}\left\{\ell^{\prime}\left(\mathbf{X}_{i}^{* T} \widehat{\beta}^{(p+a)}, Y_{i}\right)\right\}^{2} K_{h^{*}}\left(X_{i}-x_{0}\right)}{\sum_{i=1}^{n} K_{h^{*}}\left(X_{i}-x_{0}\right)},
$$

where $\widehat{\beta}^{(p+a)}=\left(\widehat{\beta}_{0}, \cdots, \widehat{\beta}_{p+a}\right)^{T}$ is the result of a $(p+a)^{t h}$-order local polynomial fit $(2.2)$ using the pilot bandwidth $h^{*}$ and $\mathbf{X}_{i}^{*}=\left(1, X_{i}-x_{0}, \cdots,\left(X_{i}-x_{0}\right)^{p+a}\right)^{T}$.

In many practical situations, it is possible to encounter over-dispersion in the first case above, i.e.

$$
\operatorname{Var}\left\{\ell^{\prime}\left(g\left(x_{0}\right), Y\right) \mid X=x_{0}\right\}=\phi V\left\{g\left(x_{0}\right)\right\}
$$

where $\phi$ is an unknown parameter. See McCullagh and Nelder (1989) for a more detailed description. In this case, we will use (4.4), instead of $V\left\{\widehat{g}_{0}\left(x_{0}\right)\right\}$ to estimate the conditional variance in (4.3).

To illustrate the idea, let use consider again the normal likelihood, in which $\ell(x, y)=-(y-x)^{2} / 2$. This implies

$$
\operatorname{Var}\left\{\ell^{\prime}\left(g\left(x_{0}\right), Y\right) \mid X=x_{0}\right\}=\operatorname{Var}\left\{Y-g\left(x_{0}\right) \mid X=x_{0}\right\}=\sigma^{2}\left(x_{0}\right)
$$

and (4.3) can be expressed as

$$
\Sigma\left(x_{0}\right)=\sigma^{2}\left(x_{0}\right) S_{n}^{-1} \bar{S}_{n} S_{n}^{-1}
$$

where $S_{n}=\mathbf{X}^{T} \mathbf{W X}$.

The right-hand side of (4.5) is exactly the approximation to the conditional variance derived by Fan and Gijbels (1995) for the locally weighted least-squares regression problem.

What does the estimator (4.4) reduce to in this special case? Here $\ell^{\prime}(u, y)=y-u$ and $\mathbf{X}_{i}^{* T} \widehat{\beta}^{(p+a)}=\widehat{Y}_{i}$, the fitted value from a local $(p+a)^{t h}$-order fit. Hence (4.4) reduces to

$$
\widehat{\sigma}^{2}\left(x_{0}\right)=\frac{\sum_{i=1}^{n}\left(Y_{i}-\widehat{Y}_{i}\right)^{2} K_{h^{*}}\left(X_{i}-x_{0}\right)}{\sum_{i=1}^{n} K_{h^{*}}\left(X_{i}-x_{0}\right)},
$$


which is asymptotically the same as the estimator for $\sigma^{2}\left(x_{0}\right)$ provided in Fan and Gijbels (1995) (see expression (2.3) in that paper or (5.2) below for a similar expression). Ruppert, Wand, Holst and Hössjer (1995) give a thorough study on the estimation of $\sigma^{2}\left(x_{0}\right)$, including the bandwidth selection and efficiency.

\section{Pilot bandwidth selector}

The estimated bias discussed in Section 3 depends on the pilot estimation of the derivatives,

$$
g^{(p+1)}\left(x_{0}\right) /(p+1) !, \cdots, g^{(p+a)}\left(x_{0}\right) /(p+a) !
$$

This in turn requires a selection of bandwidth. Also the estimation of the variance, described in the previous section, requires selection of a pilot bandwidth.

To motivate our selection procedure, let us consider the least-squares case studied in Fan and Gijbels (1995). Suppose the goal is to estimate $g^{(\nu)}(\cdot)$ using a local polynomial fit of order $p$. Let $h_{\mathrm{opt}}\left(x_{0}\right)$ be the asymptotic optimal bandwidth that minimizes the asymptotic optimal MSE

of $\widehat{\beta}_{\nu}\left(x_{0}\right)$. In the least-squares case, Fan and Gijbels (1995) define the following Residual Squares Criterion (RSC):

$$
\operatorname{RSC}\left(x_{0} ; h\right)=\widehat{\sigma}^{2}\left(x_{0}\right)\{1+(p+1) / N\}
$$

where $\widehat{\sigma}^{2}(\cdot)$ is the normalized weighted residual sum of squares after fitting locally a $p^{t h}$-order polynomial given by

$$
\widehat{\sigma}^{2}\left(x_{0}\right)=\frac{\sum_{i=1}^{n}\left(Y_{i}-\widehat{Y}_{i}\right)^{2} K_{h}\left(X_{i}-x_{0}\right)}{\operatorname{tr}(\mathbf{W})-\operatorname{tr}\left\{\left(\mathbf{X}^{T} \mathbf{W} \mathbf{X}\right)^{-1} \mathbf{X}^{T} \mathbf{W}^{2} \mathbf{X}\right\}},
$$

and $N^{-1}$ is the first diagonal element of the matrix $\left(\mathbf{X}^{T} \mathbf{W} \mathbf{X}\right)^{-1}\left(\mathbf{X}^{T} \mathbf{W}^{2} \mathbf{X}\right)\left(\mathbf{X}^{T} \mathbf{W} \mathbf{X}\right)^{-1}$. Note that $N$ in fact reflects the effective number of local data points, since $\operatorname{Var}\left\{\widehat{\beta}_{0} \mid \not X\right\} \approx \sigma^{2}\left(x_{0}\right) / N$ by (4.5). The intuition behind (5.1) is as follows. When the bandwidth $h$ is too large, the polynomial does not fit well. The bias is large and so is $\widehat{\sigma}^{2}\left(x_{0}\right)$. When the bandwidth $h$ is too small, the variance of the fit will be large and hence $N^{-1}$ will be large as well. Both factors, $\widehat{\sigma}^{2}\left(x_{0}\right)$ and $N$, are incorporated into RSC in such a way that the quantity becomes large at both extreme choices of bandwidth. It is shown in Fan and Gijbels (1995) that the minimizer of (5.1) is only a constant 
factor away from the targeted optimal bandwidth:

$$
h_{\mathrm{opt}}\left(x_{0}\right)=\operatorname{adj}_{p, \nu}(K) h_{o}\left(x_{0}\right)
$$

where $h_{o}\left(x_{0}\right)$ is the asymptotic optimal bandwidth that minimizes the main terms of the expected value of $(5.1)$, and $\operatorname{adj}_{p, \nu}(K)$ is a known constant that depends only on $K, p$ and $\nu$ (see definition below), and is tabulated in Fan and Gijbels (1995). The exact expression of this constant is as follows. Let $\mu_{j}=\int t^{j} K(t) d t$. Define the $(p+1) \times(p+1)$ matrix $S$ with the $(i+j-2)^{t h}$-moment $\mu_{i+j-2}$ of $K$ as its $(i, j)^{t h}$-element. Let $K_{\nu}^{*}(t)=\left\{\sum_{j=0}^{p} s^{\nu+1, j} t^{j}\right\} K(t)$ be the equivalent kernel, where $s^{\nu+1, j}$ is the $(\nu+1, j)^{t h}$-element of $S^{-1}$. The constants $\operatorname{adj}_{p, \nu}(K)$ are defined by

$$
\operatorname{adj}_{p, \nu}(K)=\left[\frac{(2 \nu+1) C_{p} \int K_{\nu}^{* 2}(t) d t}{(p+1-\nu)\left\{\int t^{p+1} K_{\nu}^{*}(t) d t\right\}^{2} \int K_{0}^{* 2}(t) d t}\right]^{1 /(2 p+3)},
$$

where $C_{p}=\mu_{2 p+2}-\left(\mu_{p+1}, \cdots, \mu_{2 p+1}\right) S^{-1}\left(\mu_{p+1}, \cdots, \mu_{2 p+1}\right)^{T}$.

The above criterion can also be used when the function $g(\cdot)$ is a transform of the mean regression function: $g(\cdot)=L\{\mu(\cdot)\}$ where $L$ is a link function and $\mu$ is the mean regression function. In this case, $\widehat{Y}_{i}=L^{-1}\left(\mathbf{X}_{i}^{T} \widehat{\beta}\right)$ in equation (5.2). This RSC-criterion corresponds to the approximately weighted squared errors in the domain of $g$ using weight $\left[L^{\prime}\{\mu(x)\}\right]^{-2}$.

An extension of the above idea is to regard the local likelihood problem as iterative local leastsquares problems. Given the current value $\beta_{c}$ of $\beta$, update $\beta_{c}$ via the local $p^{\text {th }}$-order polynomial regression of the working variable

$$
Z_{i}=\mathbf{X}_{i}^{T} \beta_{c}-\frac{\ell^{\prime}\left(\mathbf{X}_{i}^{T} \beta_{c}, Y_{i}\right)}{E\left\{\ell^{\prime \prime}\left(g\left(x_{0}\right), Y\right) \mid X=x_{0}\right\}}
$$

on $X_{i}$, where the conditional expectation is computed using the parameter $\beta_{c}$. The justification of this is given in the appendix. Thus, at the last step of the iteration, we can regard the local likelihood problem as a local polynomial regression problem, and use the residual squares criterion

$$
\operatorname{ERSC}\left(x_{0} ; h\right)={\widehat{\sigma_{*}}}^{2}\left(x_{0}\right)\{1+(p+1) / N\}
$$

where ${\widehat{\sigma_{*}}}^{2}\left(x_{0}\right)$ is the normalized residual sum of squares using the working variable $Z_{i}$ (compare with (5.1)). We will refer to the criterion (5.6) as the Extended Residual Squares Criterion (ERSC). The heuristic justification of this is simple. First of all, the bias of $\widehat{\beta}$ comes from the local polynomial 
approximation of $g$. Hence, it is the same for the local likelihood method as for the local leastsquares problem. Using (4.3) together with approximation (A.2),

$$
\operatorname{Var}(\widehat{\beta} \mid \not X) \approx \sigma_{*}^{2}\left(x_{0}\right) S_{n}^{-1} \bar{S}_{n} S_{n}^{-1}
$$

where

$$
\sigma_{*}^{2}\left(x_{0}\right)=\operatorname{Var}\left\{\ell^{\prime}\left(g\left(x_{0}\right), Y\right) \mid X=x_{0}\right\}\left[E\left\{\ell^{\prime \prime}\left(g\left(x_{0}\right), Y\right) \mid X=x_{0}\right\}\right]^{-2} .
$$

Comparing (4.3) with (4.5), the asymptotic variance of the local likelihood problem corresponds to that of the least-squares problem with $\sigma=\sigma_{*}$. Treating $\beta_{c}$ in (5.5) as fixed, the working variable $Z_{i}$ has the same variance structure, namely

$$
\operatorname{Var}\left(Z_{i} \mid X_{i}\right) \approx \sigma_{*}^{2}\left(x_{0}\right)
$$

We now can select the pilot bandwidth as follows. Let

$$
\widehat{h}_{p, \nu}^{*}=\arg \min _{h} \int \operatorname{ERSC}(x ; h) w(x) d x,
$$

for some given weight function $w$. Then, define the ERSC-selector as follows

$$
\widehat{h}_{p, \nu}^{\mathrm{ERSC}}=\operatorname{adj}_{p, \nu}(K) \widehat{h}_{p, \nu}^{*}
$$

ERSC in (5.7) can be replaced by RSC, (5.1), to produce a RSC-selector. As mentioned above, in the case that $g(\cdot)=L\{\mu(\cdot)\}$, the ERSC-selector with uniform weighting will be approximately the same as the RSC-selector with weight $w(x)=\left[L^{\prime}\{\mu(x)\}\right]^{-2}$. In the least-squares problem, this bandwidth selector was investigated in Fan and Gijbels (1995). It performs reasonably well, but the rate of convergence can be improved. For this reason, we only use it in the pilot stage.

\section{Bandwidth selection}

Recall that the estimation procedure consists of maximizing the local log-likelihood (2.2), leading to the estimated vector $\widehat{\beta}$. The complexity of the model is determined by the bandwidth $h$. If $h \rightarrow \infty$ then (2.2) results in a global fit of a polynomial of degree $p$. If on the other hand $h \rightarrow 0$ then we end up with interpolation of the data. Many interesting models lie between these two extreme choices. In this section we discuss data-driven choices of a constant and local variable bandwidth. 
The basic idea for bandwidth selection is very simple. First a pilot bandwidth $\widehat{h}_{p+a, p+1}^{*}$ should be selected. This can be done by either the RSC-criterion (5.1) or the ERSC-criterion (5.6). As noted at the end of Section 5, the difference is only a matter of weighting scheme. Given a pilot bandwidth $\widehat{h}_{p+a, p+1}^{*}$, we then first fit a polynomial of degree $p+a$ locally via maximizing (2.2), resulting in the estimator $\widehat{\beta}^{(p+a)}=\left(\widehat{\beta}_{0}, \cdots, \widehat{\beta}_{p+a}\right)^{T}$. With these estimated parameters we obtain the estimated bias $\widehat{B}_{p, \nu}\left(x_{0} ; h\right)$ and variance $\widehat{V}_{p, \nu}\left(x_{0} ; h\right)$ of $\widehat{\beta}_{\nu}$, which are, respectively, the $(\nu+1)^{t h}$. element of (3.3) and the $(\nu+1)^{t h}$-diagonal element of the estimated expression (4.3). An estimator for the Mean Squared Error (MSE) of $\widehat{\beta}_{\nu}$ is then given by

$$
\widehat{\operatorname{MSE}}_{p, \nu}\left(x_{0} ; h\right)=\widehat{B}_{p, \nu}^{2}\left(x_{0} ; h\right)+\widehat{V}_{p, \nu}\left(x_{0} ; h\right)
$$

This leads to the following bandwidth selector:

$$
\widehat{h}_{p, \nu}=\arg \min _{h} \int \widehat{\mathrm{MSE}}_{p, \nu}(x ; h) w(x) d x
$$

where $w(\cdot)$ is a given weight function. A common choice of $w(\cdot)$ is the indicator function of the interval where the curve $g^{(\nu)}(\cdot)$ is to be estimated.

The constant bandwidth $\widehat{h}_{p, \nu}$, which is independent of the location $x_{0}$, suffices in many applications. However, when the curve $g^{(\nu)}(\cdot)$ admits various degrees of smoothness at different locations, a variable bandwidth selector is needed in order to enhance the spatial adaptation. The basic ideas for selecting such a variable bandwidth are simple: select a bandwidth $\widehat{h}_{p, \nu}\left(x_{0}\right)$ that minimizes the locally weighted average of $\widehat{\operatorname{MSE}}_{p, \nu}(x ; h)$ around the point $x_{0}$. This average stabilizes the estimated MSE. The implementation is analogous to that discussed in Fan and Gijbels (1995) in the least-squares regression context. We omit details here.

\section{Confidence Intervals}

A confidence interval is a very important tool for understanding the sampling variability of an estimator. In the context of nonparametric function estimation, the task of constructing such an interval is difficult, due to non-negligible bias. However, with our estimated bias and variance, one can easily construct a confidence interval. 
Define

$$
\begin{aligned}
\widehat{B}_{p, \nu}^{A}\left(x_{0} ; h\right) & =\int \widehat{B}_{p, \nu}(x ; h) K_{h}\left(x-x_{0}\right) d x \\
\widehat{V}_{p, \nu}^{A}\left(x_{0} ; h\right) & =\int \widehat{V}_{p, \nu}(x ; h) K_{h}\left(x-x_{0}\right) d x
\end{aligned}
$$

These two quantities are just local weighted averages of the estimated bias and variance, respectively. Note that the estimated bias involves the estimation of higher order derivative curves, whose estimation can be unstable. The purpose of the average is to stabilize the estimated bias and variance function, and to prevent them from abrupt change. The same bandwidth as used for (2.2) is used here, but a different bandwidth could also be employed. This was done primarily for simplicity of implementation, but Brockmann, Gasser, and Herrmann (1993) have used this amount of smoothing for a local bias estimate, which produced a location dependent bandwidth in traditional kernel based regression. This adaptive method has both good asymptotic and practical performance.

The local maximum likelihood estimator is usually asymptotically normal. In the context of generalized linear models, this has been shown by Fan, Heckman and Wand (1995) and in the context of hazards regression by Fan, Gijbels and King (1997). By invoking the asymptotic normality, we construct the pointwise confidence interval as follows. With approximately $1-\alpha$ coverage probability, the unknown function $g^{(\nu)}\left(x_{0}\right)$ falls in the random interval

$$
\widehat{g}_{\nu}\left(x_{0}\right)-\widehat{B}_{p, \nu}^{A}\left(x_{0} ; h\right) \pm \Phi^{-1}(1-\alpha / 2)\left\{\widehat{V}_{p, \nu}^{A}\left(x_{0} ; h\right)\right\}^{1 / 2} .
$$

The coverage probability of the confidence interval (7.3) can converge slowly to the nominal level $1-\alpha$. There are two reasons for that. One is that the number of data points used to estimate $g^{(\nu)}\left(x_{0}\right)$ can be much smaller than $n$ and the other is that the bias can possibly be non-negligible. Nevertheless, Figures $8.1(\mathrm{~d}), 8.2(\mathrm{~d})$ and $8.3(\mathrm{~d})$ report reasonably satisfactory coverage probability in our simulation studies.

In our implemention, the confidence interval (7.3) is constructed based on the estimated optimal bandwidth. Technically, the asymptotic normality still holds with such a data-driven bandwidth owing to the tightness of the stochastic process indexed by bandwidth $h$ (See Müller and Stadtmüller (1987) for technical arguments in a simpler setup). With the optimal bandwidth, the estimator 
$\widehat{g}^{(\nu)}\left(x_{0}\right)$ has smaller asymptotic MISE than any other choice of bandwidth and hence the confidence interval is expected to be tighter.

An alternative approach for constructing confidence intervals is to undersmooth the estimated curve in such a way that the bias of the estimator is negligible. While this idea is simple and useful, it has a few potential shortcomings: It is hard to know how small the bandwidth will need to be to make the bias negligible; with an undersmoothed estimator, the variance will be larger and hence the confidence intervals will tend to be wider; the asymptotic normality for the undersmoothed estimator tends to actualize itself more slowly because there are fewer local data points.

For constructing simultaneous confidence bands, one can use the raw materials given in (7.1) and (7.2) and the ideas given in Eubank and Speckman (1993).

\section{Applications to logistic regression}

We have illustrated the key idea of this paper in the context of the least-squares regression problem. Extensive simulations in Fan and Gijbels (1995) indicate good performance of the resulting procedure. We now consider nonparametric logistic regression to further clarify the idea. The method can readily be applied to other likelihood models such as those based on the Poisson and Gamma distributions.

\subsection{Illustration}

We assume that the data, $\left(X_{i}, Y_{i}\right)$, are i.i.d. and that the conditional distribution of $Y_{i}$ given $X_{i}$ is a Bernoulli distribution:

$$
P\left(Y_{i}=1 \mid X_{i}=x\right)=p(x) ; \quad P\left(Y_{i}=0 \mid X_{i}=x\right)=1-p(x) \equiv q(x) .
$$

In this case, the mean regression function is $p(x)=E(Y \mid X=x)$. The parameter of interest is

$$
g(x)=\operatorname{logit}(p(x))=\log \frac{p(x)}{1-p(x)} .
$$

In this nonparametric regression context, estimating $p(\cdot)$ is equivalent to estimating $g(\cdot)$. However, we prefer working on the logit domain, since the log-likelihood is concave, and the logistic linear 
regression model corresponds to our case with $h=\infty$. See Fan, Heckman and Wand (1995) for more detailed arguments.

The log-likelihood is determined via

$$
\ell(g(X), Y)=\log \left\{p(X)^{Y} q(X)^{1-Y}\right\}=Y g(X)-\log [1+\exp \{g(X)\}]
$$

Thus, $\ell^{\prime}(u, y)=y-e^{u} /\left(1+e^{u}\right)$ and

$$
E\left\{\ell^{\prime}(g(X), Y) \mid X\right\}=0, \quad \operatorname{Var}\left\{\ell^{\prime}(g(X), Y) \mid X\right\}=p(X) q(X)
$$

For this case, we have

$$
L_{p}^{*}\left(\beta ; h, x_{0}\right)=\sum_{i=1}^{n}\left[Y_{i}\left(\mathbf{X}_{i}^{T} \beta+r_{i}\right)-\log \left\{1+\exp \left(\mathbf{X}_{i}^{T} \beta+r_{i}\right)\right\}\right] K_{h}\left(X_{i}-x_{0}\right) .
$$

The local likelihood $L_{p}$ corresponds to $L_{p}^{*}$ with $r_{i} \equiv 0$. Define

$$
p_{i}^{*}=\frac{\exp \left(\mathbf{X}_{i}^{T} \beta+r_{i}\right)}{1+\exp \left(\mathbf{X}_{i}^{T} \beta+r_{i}\right)}, \quad \text { and } \quad p_{i}=\frac{\exp \left(\mathbf{X}_{i}^{T} \beta\right)}{1+\exp \left(\mathbf{X}_{i}^{T} \beta\right)}
$$

Then, simple algebra shows that

$$
\begin{aligned}
L_{p}^{* \prime}\left(\beta ; h, x_{0}\right) & =\sum_{i=1}^{n}\left(Y_{i}-p_{i}^{*}\right) \mathbf{X}_{i} K_{h}\left(X_{i}-x_{0}\right) \\
L_{p}^{* \prime \prime}\left(\beta ; h, x_{0}\right) & =-\sum_{i=1}^{n} p_{i}^{*}\left(1-p_{i}^{*}\right) \mathbf{X}_{i} \mathbf{X}_{i}^{T} K_{h}\left(X_{i}-x_{0}\right) .
\end{aligned}
$$

The approximated bias vector and variance matrix are now easily obtained, and are given by

$$
\begin{aligned}
\hat{b}_{p}\left(x_{0}\right) & =\left\{L_{p}^{* \prime \prime}\left(\widehat{\beta} ; h, x_{0}\right)\right\}^{-1} L_{p}^{* \prime}\left(\widehat{\beta} ; h, x_{0}\right) \\
\Sigma\left(x_{0}\right) & =p\left(x_{0}\right) q\left(x_{0}\right)\left\{\sum_{i=1}^{n} p_{i} q_{i} \mathbf{X}_{i} \mathbf{X}_{i}^{T} K_{h}\left(X_{i}-x_{0}\right)\right\}^{-1}\left(\mathbf{X}^{T} \mathbf{W}^{2} \mathbf{X}\right)\left\{\sum_{i=1}^{n} p_{i} q_{i} \mathbf{X}_{i} \mathbf{X}_{i}^{T} K_{h}\left(X_{i}-x_{0}\right)\right\}^{-1},
\end{aligned}
$$

where $q_{i}=1-p_{i}$.

It can also be shown that

$$
\operatorname{ERSC}\left(x_{0} ; h_{0}\right)=2\{1+(p+1) / N\} \sum_{i=1}^{n}\left[Y_{i} \log \left(Y_{i} / \widehat{p}_{i}\right)+\left(1-Y_{i}\right) \log \left\{\left(1-Y_{i}\right) /\left(1-\widehat{p}_{i}\right)\right\}\right] K_{h}\left(X_{i}-x_{0}\right),
$$

where $\widehat{p}_{i}$ is given by (8.2) with $\beta$ being estimated. Minimizing the average of ERSC gives a pilot bandwidth selector as discussed in Section 5 . 


\subsection{Implementation}

In the context of logistic regression, the two stage bandwidth selector was tested on simulated data from a variety of target curves. The Epanechnikov kernel

$$
K(u)=\frac{3}{4}\left(1-u^{2}\right) I_{[-1,1]}(u)
$$

and order $p=1$ were used for estimating $g$ at a set of equally spaced grid points $x_{1}, \ldots, x_{\mathrm{nbin}}$. The RSC-criterion was used to choose the pilot bandwidth with a $p=3$ order fit for estimating the curvature $g^{\prime \prime}$. The sum

$$
\operatorname{ARSC}\left(h_{j}\right)=\sum_{i=\tau}^{\text {nbin }-\tau} \operatorname{RSC}\left(x_{i} ; h_{j}\right),
$$

where $\tau$ is the largest integer less than $(0.05 \mathrm{nbin})$, was computed on a multiplicative grid of bandwidths $h_{j}=C^{j} h_{\min }$. Restricting the sum helps to reduce boundary effects. At the boundaries, RSC and estimated derivatives can be too large due to numerical instabilities and scarcity of data.

The implementation of our idea is somewhat tricky since it involves iterative solution. For small values of $h$, the solution may not even exist since locally one may get a set of all zeros or ones. To avoid such a difficulty, we only consider the bandwidths that are large enough so that the $\widehat{\beta}^{\prime} s$ are defined. To this goal, let $l_{\text {runs }}$ be the maximum span among lengths of runs of ones or zeros. For instance, if $X_{(1)}, \ldots, X_{(n)}$ are the order statistics of the $X$ sample and the bivariate data are sorted according to the $X$ sample, then $X_{(k)}-X_{(j)}$ is the length of a run of ones provided

$$
Y_{j-1}=0, Y_{j}=1, Y_{j+1}=1, \ldots, Y_{k}=1, Y_{k+1}=0
$$

The choice of the minimum value of $h$ was given by

$$
h_{\min }=\left(1.25 l_{\text {runs }}+6 \Delta_{g}\right) / 2
$$

where $\Delta_{g}$ is the grid spacing. The maximum value of the bandwidth was set at

$$
h_{\max }=\max _{j}\left\{C^{j} h_{\min }: C^{j} h_{\min }<\left(x_{\text {nbin }}-x_{1}\right) / 2\right\}
$$

with $C=1.1$. 


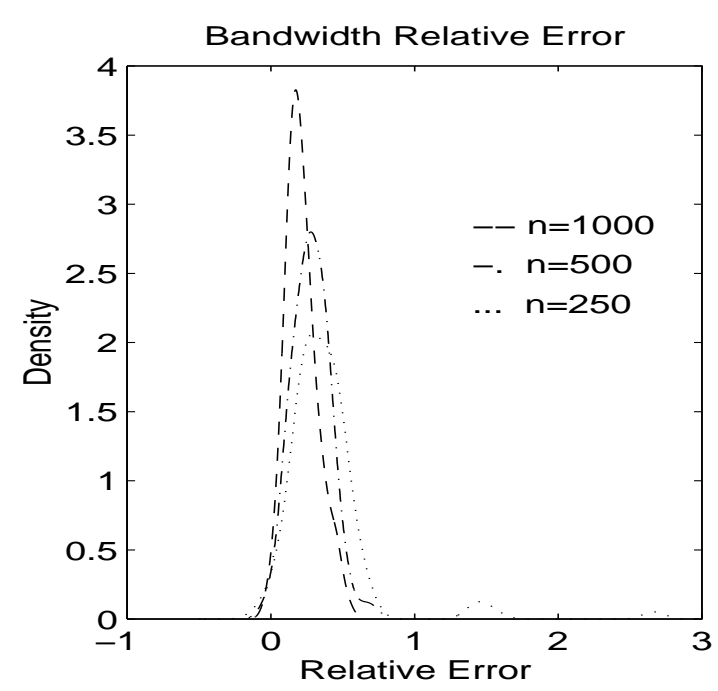

(a)

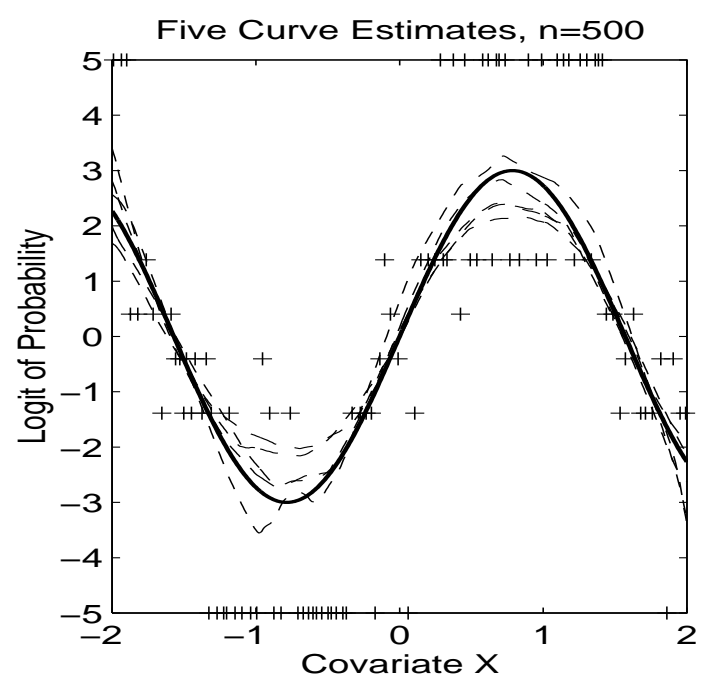

(c)

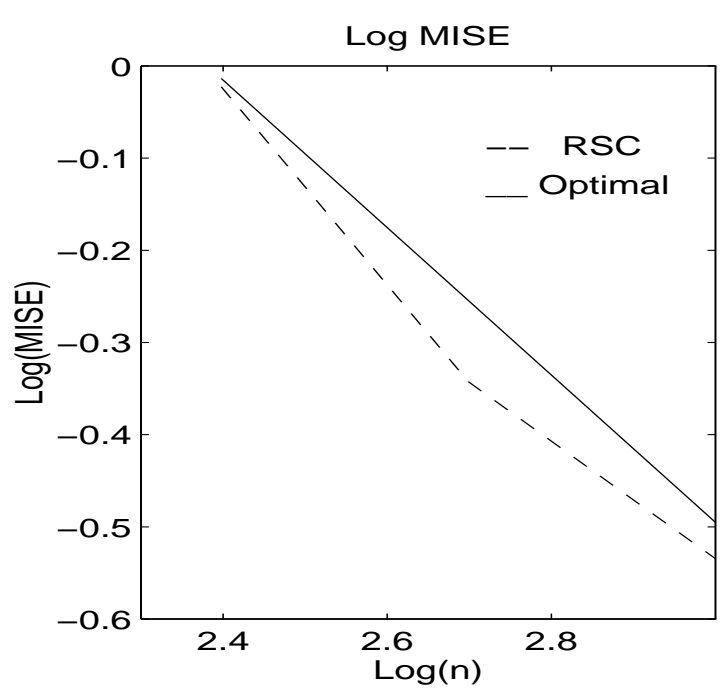

(b)

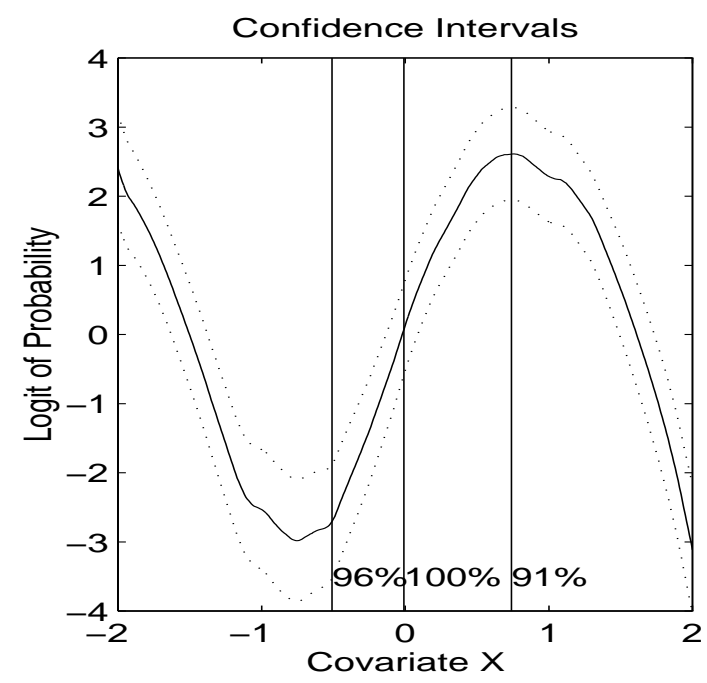

(d)

Figure 8.1: Example 1. (a) Kernel Density Estimate of bandwidth relative errors for three sample sizes. (b) Base $10 \mathrm{log}$ of MISE versus sample size. The asymptotically optimal MISE is indicated by the solid curve. (c) Five sample curve estimates with true curve (solid line) and sample logits $(+)$ using five contiguous observations. (d) Sample curve estimate (solid line) with confidence interval (dashed line) and the percentage of confidence intervals containing the true curve at three separate points indicated with vertical lines.

\subsection{Simulation Results}

Results for three underlying target curves on the logit scale are presented. Sample sizes of $n=$ 250, 500, 1000 were used. The design is from a uniform distribution on $[-2,2]$. In other words, the marginal distribution for $X$ is uniform on $[-2,2]$. The logit transform of the conditional probability 
$Y=1$ is given by

$$
\begin{array}{ll}
\text { Example 1. } & g(x)=3 \sin (2 x) \\
\text { Example 2. } & \left.g(x)=7\left[\exp \left\{-(x+1)^{2}\right\}+\exp \left\{-(x-1)^{2}\right)\right\}\right]-5.5 \\
\text { Example 3. } & g(x)=2-x^{2} .
\end{array}
$$

These curves appear as the thick line in part (c) of the following figures. Only 100 simulations were performed for a given target curve at a given sample size. This was a practical constraint due to the fact that it takes approximately 20 minutes to compute the two stage bandwidth using a prototype implementation in Matlab on a Sparc 10 station. This can be improved upon by at least a factor of 10, if a lower level language such as $\mathrm{C}$ is used. The asymptotically optimal bandwidth from Fan, Heckman and Wand (1995)

$$
h_{\mathrm{opt}}=\left\{\frac{\int K(u)^{2} d u \int \operatorname{Var}(Y \mid X=x) L^{\prime}(\mu(x))^{2} w(x) / f_{X}(x) d x}{n\left(\int u^{2} K(u) d u\right)^{2} \int g^{\prime \prime}(x)^{2} w(x) d x}\right\}^{1 / 5}
$$

with $w$ taken to be the indicator function on $[-2,2]$ was used to judge performance.

\begin{tabular}{||c|cc|cc|cc||}
\hline & Example 1 & & Example 2 & & \multicolumn{2}{|c||}{ Example 3 } \\
$n$ & $h_{\text {opt }}$ & $h_{\text {MedISE }}$ & $h_{\text {opt }}$ & $h_{\text {MedISE }}$ & $h_{\text {opt }}$ & $h_{\text {MedISE }}$ \\
\hline 250 & 0.53 & 0.64 & 0.48 & 0.53 & 0.83 & 1.04 \\
500 & 0.46 & 0.59 & 0.42 & 0.47 & 0.72 & 0.86 \\
1000 & 0.40 & 0.44 & 0.36 & 0.40 & 0.63 & 0.78 \\
\hline
\end{tabular}

Table 1: Comparison of asymptotic and small sample optimal bandwidths, where the asymptotic optimal bandwidth is denoted by $h_{\text {opt }}$ and the median integrated squared error optimal bandwidth is denoted by $h_{\text {MedISE }}$. The MedISE optimal bandwidth is based on the unconditional expectation computed by simulation.

Both the conditional and unconditional Mean Integrated Squared Error (MISE) of the local maximum likelihood estimator are not mathematically defined. For any fixed bandwidth, there is positive probability that all the data in the "window" is either one or zero. In this case, the maximizer of (2.2) is infinite. Thus, the MISE-based optimal bandwidths are not properly defined. In contrast, the optimal bandwidths based on the Median Integrated Squared Error (MedISE) are properly defined and this avoids the technical difficulty of the MISE. In order to assess the appropriateness of $h_{\mathrm{opt}}$ for judging finite sample performance, the MedISE was computed by simulation 


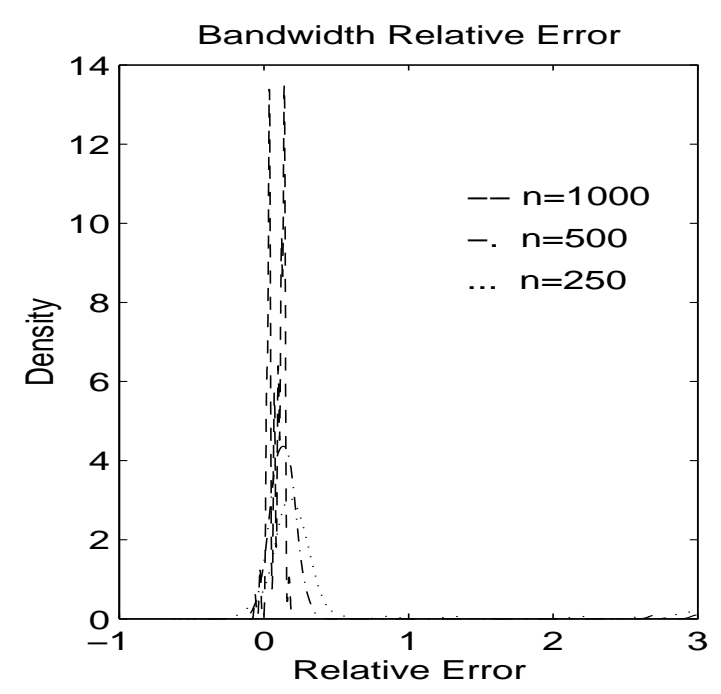

(a)

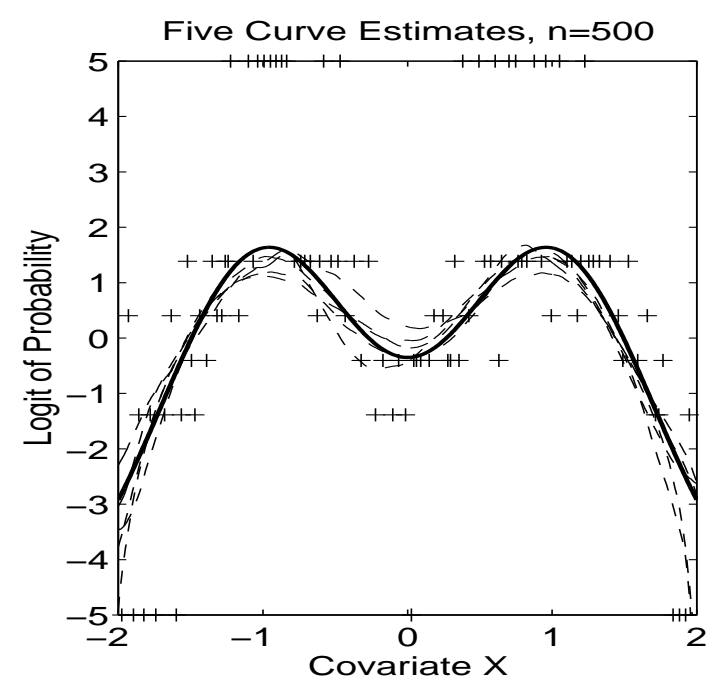

(c)

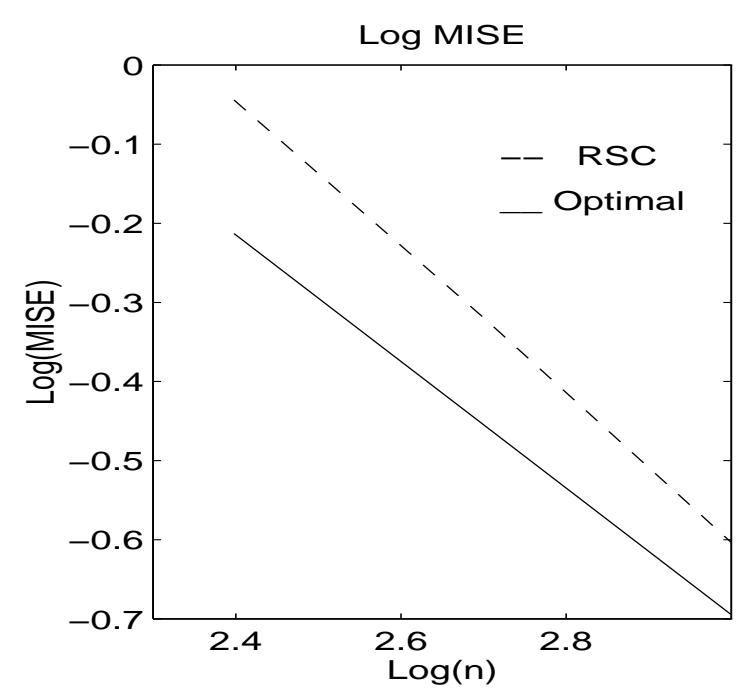

(b)

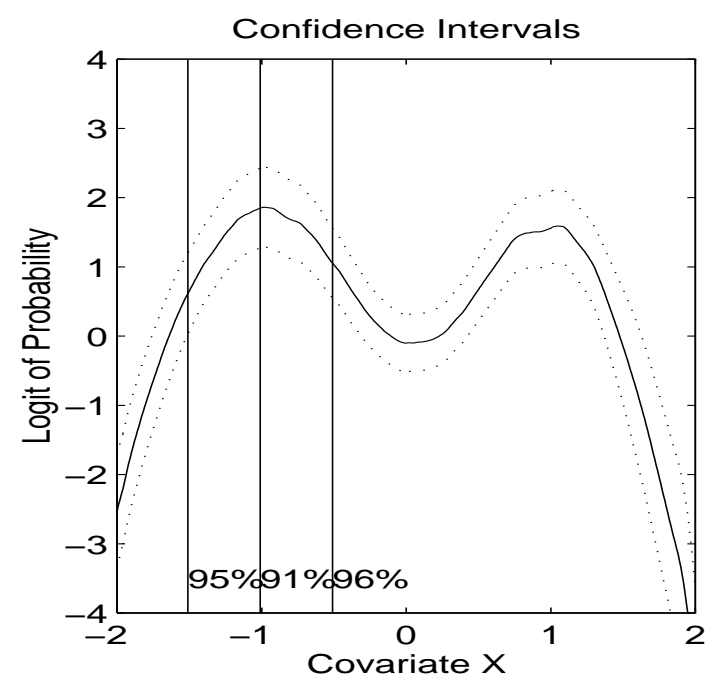

(d)

Figure 8.2: Example 2. (a) Kernel Density Estimate of bandwidth relative errors for three sample sizes. (b) Base $10 \mathrm{log}$ of MISE versus sample size. The asymptotically optimal MISE is indicated by the solid curve. (c) Five sample curve estimates with true curve (solid line) and sample logits (+) using five contiguous observations. (d) Sample curve estimate (solid line) with confidence interval (dashed line) and the percentage of confidence intervals containing the true curve at three separate points indicated with vertical lines.

on the bandwidth grid, $h_{j}=C^{j} h_{\min }$. The simulated MedISE was a convex function of the bandwidth, $h$, for all three examples and the minimizing value of $h$ for each example appears in Table 1. The simulated MedISE was based on 400 curve estimates and the $95 \%$ error margin was typically within $10 \%$ of the minimizing value and never more than $20 \%$. The closeness of $h_{\text {opt }}$ to the MedISE optimal bandwidth makes it appropriate for a bench mark for performance. 


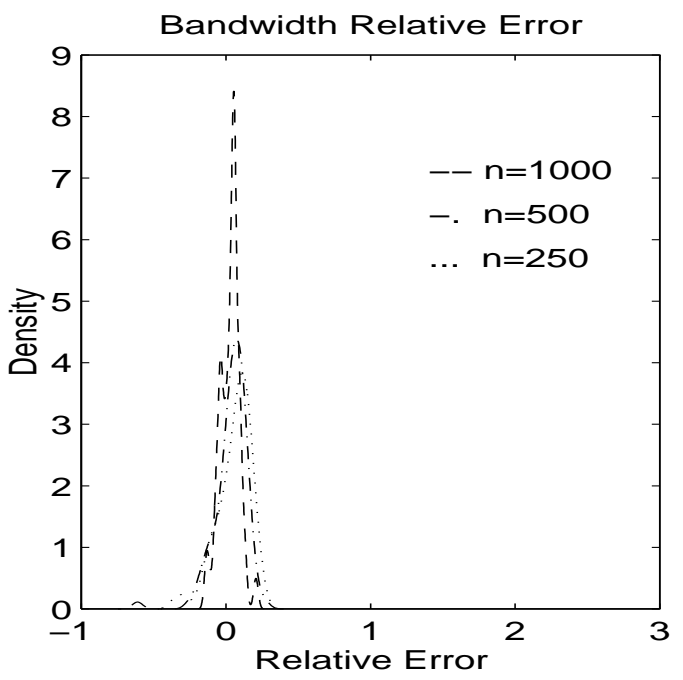

(a)

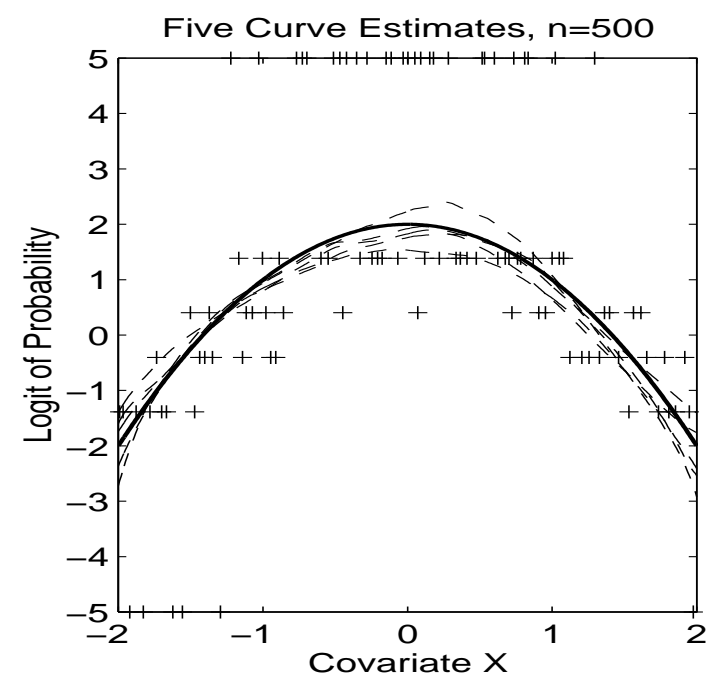

(c)

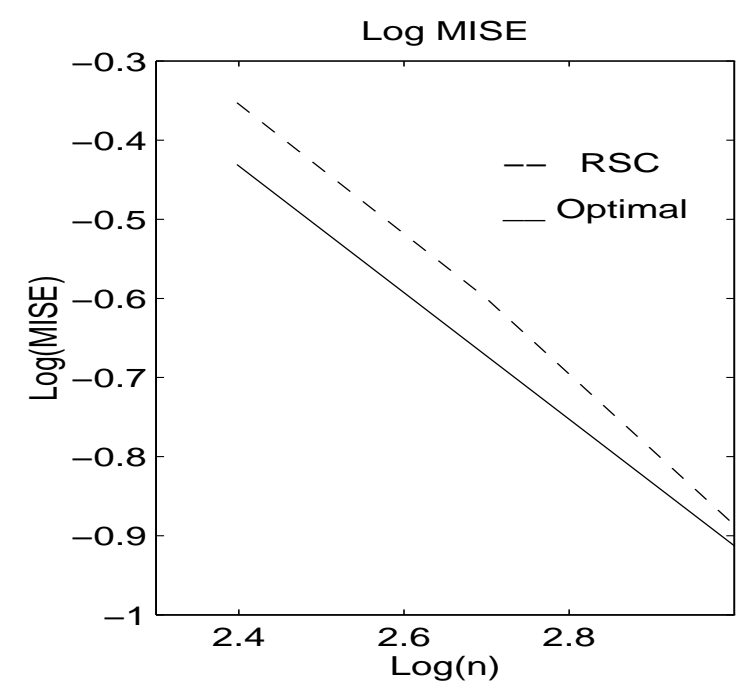

(b)

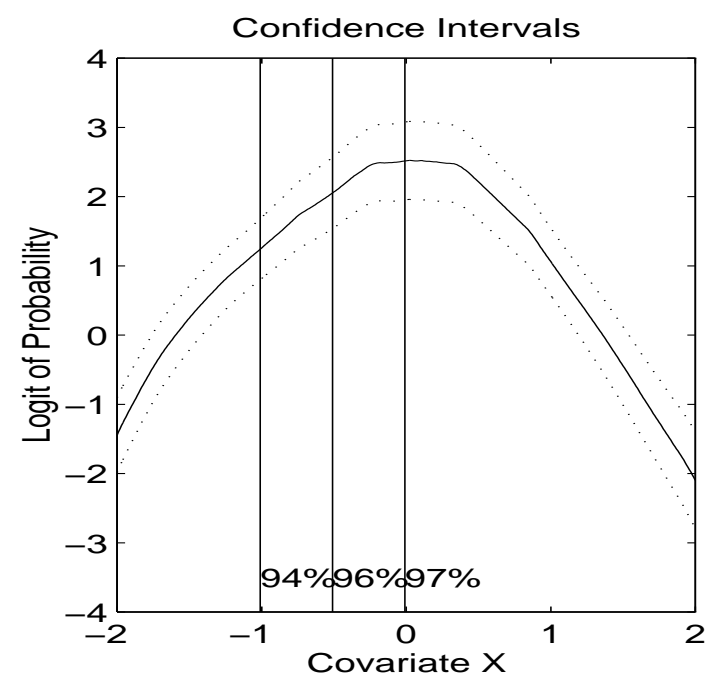

(d)

Figure 8.3: Example 3. (a) Kernel Density Estimate of bandwidth relative errors for three sample sizes. (b) Base $10 \mathrm{log}$ of MISE versus sample size. The asymptotically optimal MISE is indicated by the solid curve. (c) Five sample curve estimates with true curve (solid line) and sample logits (+) using five contiguous observations. (d) Sample curve estimate (solid line) with confidence interval (dashed line) and the percentage of confidence intervals containing the true curve at three separate points indicated with vertical lines.

The relative error of the two stage bandwidth $\widehat{h}$ is computed as $\left(\widehat{h}-h_{\mathrm{opt}}\right) / h_{\mathrm{opt}}$. In addition to relative error, the median of the integrated squared errors of the 100 curve estimates was taken and the percentage of the 100 confidence intervals that contained the true curve at a given point was also computed. The results are summarized in Figures 8.1, 8.2 and 8.3. Note that the standard error for the sample coverage is $(0.05 * 0.95 / 100)^{1 / 2}=2.18 \%$. 
Observe that the relative errors become more and more concentrated near zero as $n$ increases. There are a few bandwidths that appear to have quite large relative errors but the number of large errors decreases with $n$. From part (b), the MISE becomes closer to the asymptotically optimal MISE as $n$ increases. Part (c) indicates the difficulty in estimating these curves by the spread of the sample logits based on five observations. The values of these logits were truncated at \pm 5 and some were actually infinite. These large sample logits are indicated by the plus marks along the axes. Details of computing sample logits are as follows: Group the data points according to their covariate values so that each group consists of 5 data points. For each group, compute the sample proportion of ones and do a logit transform of it. This sample logit is then plotted against mean covariate values of its corresponding group. The sample curve estimates indicate that the estimator performs quite well. The pointwise confidence intervals also have good performance. Figures 8.1 and 8.2 indicate some difficulty near the peaks but this difficulty is expected because the bias can be quite large near sharp peaks. The curve estimate in the confidence interval is the bias corrected curve estimate $\widehat{g}_{0}(x)-\widehat{B}_{1,0}^{A}(x ; h)($ see $(7.3))$.

\section{Concluding Remarks}

We have laid out a versatile approach for nonparametric smoothing, bandwidth selection and confidence interval construction. The purpose of this article is to indicate that there is a unified approach to nonparametric smoothing. We have only extensively tested the idea in the context of least-squares and in a few other cases. Further studies are needed to test the approach in other contexts. We hope this article will stimulate future research on this topic.

Acknowledgement. Jianqing Fan was supported by NSF Grant DMS9504414 and NSA grant 961-0015. Irène Gijbels was supported by 'Projet d'Actions de Recherche Concertées' (No. 93/98 164), an FNRS-grant (No. 1.5.001.95F) and the European Human Capital and Mobility Programme (CHRX-CT94-0693). The authors thank an associate editor and a referee for their insightful comments that led to improving the presentation. 


\section{REFERENCES}

Brockmann, M., Gasser, T. and Herrmann, E. (1993). Locally adaptive bandwidth choice for kernel regression estimators. Jour. Amer. Statist. Assoc., 88, 1302-1309.

Carroll, R.J., Ruppert, D. and Welsh, A.H. (1996). Nonparametric estimation via local estimating equations, Unpublished manuscript.

Cleveland, W.S. (1979). Robust locally weighted regression and smoothing scatterplots. Jour. Amer. Statist. Assoc., 74, 829-836.

Copas, J.B. (1995). Local likelihood based on kernel censoring. Jour. Royal Statist. Society Series $\mathrm{B}, \mathbf{5 7}, 221-235$.

Cox, D.R. (1972). Regression models and life-tables (with discussion). Jour. Royal Statist. Society Series B, 4, 187-220.

Eubank, R. and Speckman, P. (1993). Confidence Bands in Nonparametric Regression. Jour. Amer. Statist. Assoc., 88, 1287-1301.

Fan, J. and Gijbels, I. (1995). Data-driven bandwidth selection in local polynomial fitting: variable bandwidth and spatial adaptation. Jour. Royal Statist. Society Series B, 57, 371-394.

Fan, J., Gijbels, I. and King, M. (1997). Local likelihood and local partial likelihood in hazard regression. Ann. Statist., 25, 1661-1690.

Fan, J. , Heckman, N.E. and Wand, M.P. (1995). Local polynomial kernel regression for generalized linear models and quasilikelihood functions. Jour. Amer. Statist. Assoc., 90, 141-150.

Friedman, J.H. and Stuetzle, W. (1981). Projection Pursuit Regression. Jour. Amer. Statist. Assoc., 76, 817-823.

Hjort, N.L. (1991). Semiparametric estimation of parametric hazard rates. In Survival Analysis: State of the Art, Kluwer, Dordrecht, 211-236. Proceedings of the NATO Advanced Study Workshop on Survival Analysis and Related Topics, eds. P.S. Goel and J.P. Klein.

Hjort, N.L. (1995). Dynamic likelihood hazard rate estimation. Biometrika, to appear.

Hjort, N.L. and Glad, I.K. (1995). Nonparametric density estimation with a parametric start. Ann. Statist., 23, 882-904.

Hjort, N.L. and Jones, M.C. (1996). Locally parametric nonparametric density estimation. Ann. Statist., 24, 1619-1647.

Jones, M.C. (1994). On close relations of local likelihood density estimation. Manuscript.

Loader, C.R. (1996). Local likelihood density estimation. Ann. Statist., 24, 1602-1618.

McCullagh, P. and Nelder, J.A. (1989). Generalized Linear Models. Second Edition. Chapman and Hall, London.

Müller, H.-G. and Stadtmüller, U. (1987). Variable bandwidth kernel estimators of regression curves. Ann. Statist., 15, 182-201. 
Nelder, J.A. and Wedderburn, R.W.M. (1972). Generalized linear models. Jour. Royal Statist. Society Series A, 135, 370-384.

Ruppert, D. (1995). Empirical-bias bandwidths for local polynomial nonparametric regression and density estimation. Technical Report \#1137, School of Operations Research and Industrial Engineering, Cornell University, Ithaca, New York.

Ruppert, D., Wand, M.P., Holst, U. and Hössjer, O. (1995). Local polynomial variance function estimation. Unpublished manuscript.

Staniswalis, J.G. (1989). The kernel estimate of a regression function in likelihood-based models. Jour. Amer. Statist. Assoc., 84, 276-283.

Stone, C.J. (1977). Consistent Nonparametric Regression. Ann. Statist., 5, 595-645.

Stone, C.J. (1980). Optimal rates of convergence for nonparametric estimators. Ann. Statist., 8, $1348-1360$.

Tibshirani, R. and Hastie, T. (1987). Local likelihood estimation. Jour. Amer. Statist. Assoc., 82, 559-567.

\section{Appendix}

Proof of (5.5). The Fisher scoring method for computing the local maximum likelihood estimator is to update $\widehat{\beta}$ via

$$
\widehat{\beta}=\beta_{0}-\left[E\left\{L_{p}^{\prime \prime}\left(\beta_{0} ; h, x_{0}\right) \mid \not X\right\}\right]^{-1} L_{p}^{\prime}\left(\beta_{0} ; h, x_{0}\right)
$$

Using the continuity assumption of the conditional expectation, it follows that

$$
\begin{aligned}
E\left\{L_{p}^{\prime \prime}\left(\beta_{0} ; h, x_{0}\right) \mid \not X\right\} & =\sum_{i=1}^{n} E\left\{\ell^{\prime \prime}\left(\mathbf{X}_{i}^{T} \beta_{0}, Y_{i}\right) \mid X_{i}\right\} \mathbf{X}_{i} \mathbf{X}_{i}^{T} K_{h}\left(X_{i}-x_{0}\right) \\
& \approx E\left\{\ell^{\prime \prime}\left(g\left(x_{0}\right), Y\right) \mid X=x_{0}\right\} \sum_{i=1}^{n} \mathbf{X}_{i} \mathbf{X}_{i}^{T} K_{h}\left(X_{i}-x_{0}\right)
\end{aligned}
$$

Note that even though the computational expectation is computed approximately, the algorithm is exact, namely it converges to the local MLE even when we use the approximated Hessian matrix (A.2). Therefore, substituting (A.2) into (A.1) and using (2.2), we have

$$
\widehat{\beta}=\left[\sum_{i=1}^{n} \mathbf{X}_{i} \mathbf{X}_{i}^{T} K_{h}\left(X_{i}-x_{0}\right)\right]^{-1} \sum_{i=1}^{n} Z_{i} \mathbf{X}_{i} K_{h}\left(X_{i}-x_{0}\right)
$$

namely, $\widehat{\beta}$ is obtained via regressing $Z_{i}$ on $X_{i}$ using locally the polynomial of order $p$. 